\title{
Diameter and Radius in the Manhattan Metric*
}

\author{
D. Z. Du and D. J. Kleitman
}

Mathematics Department, Massachusetts Institute of Technology, Cambridge, MA 02139, USA

\begin{abstract}
We investigate maximum size sets of lattice points with a given diameter, $d$, within a given rectilinearly bounded finite region $R$ in $n$ dimensions, under the Manhattan or $L 1$ metric. We show that when the length of $R$ in each dimension is an odd integer (as, for example, the $n$-cube) there is, for every integer $d$, a maximum size set having radius $d / 2$ about some center, though the center need not be a lattice point.

Similar results are obtained when $R$ has even length in some dimensions, except for a set of $d$ values whose cardinality is one less than the number of dimensions in which $R$ has even length. This question is still open for these values.
\end{abstract}

\section{Introduction}

We are concerned with lattice points in a set $R$ which can be described as integer coordinate $n$-component vectors, with prescribed upper and lower bounds on each coordinate. The distance between two such vectors is the sum of the magnitudes of their coordinate differences.

The $n$-cube is an example of such a set, for which each of the $n$ components is either zero or one. The $n$-cube has the property that for $d=2 r, d<n$, the largest possible size of a set of its elements that has diameter $d$ is the number of its elements at or within radius $r$ of one of its elements [1]. The corresponding statement fails for $d$ odd or for $d=n$ in the $n$-cube. However, for any integral $d$, the largest possible size of a subset of diameter $d$ is the number of elements at or within a distance $d / 2$ of some center, in the $n$-cube.

A subset of $R$ having diameter $d$ that is of maximum size among such sets is called a maximum $d$ set.

\footnotetext{
* This research was supported in part by AF Grant OSR-86-0078 and NSF Grant DMS-86-06225.
} 
Erdös suggested the general question: for what $d$ values and what more general-sized regions $R$ is there a maximum $d$ set of radius $d / 2$ and center in $R$ ?

Kleitman and Fellows [2] found a partial answer to Erdös's question, showing that, for $d$ even and below a certain bound, that statement holds. In particular, let $d_{j}$ be the largest coordinate difference in the $j$ th component among members of $R$, and let $c_{j}=\left[\left(d_{j}+1\right) / 2\right]$ (the smallest integer at least $\left.d_{j} / 2\right)$. They showed that if $d$ is even and $d<\sum_{j} c_{j}=b(R)$, then the set of elements of $R$ at or within $d / 2$ of one of its "central" elements is a maximum $d$ set.

Kleitman and Fellows's argument was rather convoluted. The quest for a simpler argument led them to the following:

Conjecture. For every $R$ having independent bounds on each coordinate, and every integer $d$, there is a set of radius $d / 2$ that is a maximum $d$ set.

If this conjecture is true, then we can prove Kleitman and Fellows's results, and any possible strengthenings of it by comparing the number of points at or within radius $d / 2$ of all possible centers. When an integer coordinate center has a largest possible radius $d / 2$ set around it, then Erdös's question has a positive answer.

It is the purpose of this note to provide a partial verification of this conjecture. Let $e(R)$ be the number of components for which $d_{j}$ is an even integer. We show that the conjecture holds except possibly for the consecutive $e(R)-1$ values of $d$ from $b(R)$ up. Thus, if $e(R)<2$ the conjecture is completely resolved.

Unfortunately, this result does not really provide a simplified proof of the Kleitman-Fellows theorem, for it requires the mechanisms and methods used by them in its proof. Thus, instead of finding a shortcut to their theorem, we show that their methods can be extended to this stronger statement.

The Kleitman-Fellows theorem in fact proves this conjecture for $d$ even and below $b(R)$. We proceed by finding a way to adapt Kleitman and Fellows's methods to deal with odd $d$ and with $d$ above $b(R)+r-1$.

In the next section we review the Kleitman-Fellows result, and the basis for the induction used. We then describe and prove the new statements. Finally, we prove it for $r=0$ and then we prove the general result.

\section{Preliminaries, Complementarity and $n$-Cube Lemmas, and Results}

The Kleitman-Fellows argument was based upon four observations:

1. In the $n$-cube there is a maximum $d$ set of radius $d / 2$ about an element of $R$ for all even $d$ with $d<n[1]$.

2. When $d$ is even, $d=b(R)-1$, and $e(R)=0$, the maximum cardinality of a $d$ diameter set is half the cardinality of $R$, and there are radius $d / 2$ sets having this cardinality.

3. We can prove the result inductively for $d$ even, $d<b(R)-1, R$ not the $n$-cube, and $e(R)=0$ essentially by separating the two outer values of some coordinate from the rest. 
4. We can extend the result when $e(R)>0$ by separating a single outer value of an appropriate coordinate from the rest.

We obtain our results here by extending these statements to general integral d. The results are as follows:

Lemma 1. In the n-cube, when $d$ is odd and $d<n$, there is a maximum d set of radius $d / 2$ about a point midway between two "adjacent" elements of $R$ (where adjacent here means differing by 1 in exactly one component). When $d=n$ the only maximum $d$ set is $R$ itself; which has radius $d / 2$ from the "center" of the cube.

We define a central element of $R$ to be an element that is closest to the geometric center of $R$ among all its elements. In the $n$-cube, for example, all elements are central.

Lemma 2. When $e(R)=0$ and $d=b(R)-1$, a maximum d set can have cardinality at most half that of $R$. And if $c$ is any point within the convex hull of the central elements of $R$, having half integral distance to each element of $R$, then when $d$ is odd, the elements at or within distance $d / 2$ of $c$ has cardinality half that of $R$.

Assume that the elements of $R$ are restricted in their $j$ th component, $x_{j}$, by $-c_{j}<x_{j} \leq c_{j}$. Let the allowed $j$ th coordinates be ordered as follows: $0,1,-1,2$, $-2, \ldots,-c_{j}+1, c_{j}$ (and finally $-c_{j}$ if that value were allowed). If, for a subset $S$ of $R$, for every $j$ and every set of values of all coordinates but the $j$ th, the elements of $S$ with those values have an initial segment of this order as values of their $j$ th coordinate, then we call $S$ canonical.

Lemma 3. There is a canonical maximum $d$ set for every $d$ and $R$.

Theorem 4. There is a radius $d / 2$ maximum $d$ set if $e(R)=0$. The center is a central lattice point if $d$ is even $d<b(R)$, displaced by $\frac{1}{2}$ in one coordinate from $a$ central lattice point if $d$ is odd and $d<b(R)$, and the center of $R$ or displaced by $\frac{1}{2}$ in one coordinate from the center of $R$ depending on the parity of $d-n$ if $d \geq b(R)$.

Theorem 5. In general there is a radius $d / 2$ maximum $d$ set if $d<b(R)$ or $d \geq b(R)+e(R)-1$. The center is as in Theorem 4 .

The three lemmas used here are very similar to results proven by Kleitman and Fellows and we only sketch their proofs here.

Proof of Lemma 1. The result is obvious for $n=1, d=n$, or $d=n-1$. (In the last of these a $d$ diameter set can contain at most one of any complementary pair of elements of $R$, and the indicated set contains one of every such pair.)

For all other $n$ and $d$ we can use the "pushing" argument and induction on $n$ in the odd case here, exactly as argued in the even case in [1]. Details are identical and are omitted here. 
Proof of Lemma 2. When $r=0$ we can define a complementation operation on vectors in $R$ by its action on each component, as follows:

$$
C\left(x_{j}\right)=x_{j}+c_{j}\left(\bmod 2 c_{j}\right) .
$$

The distance between complementary vectors is then $b(R)$. Therefore at most one of any complementary pair is in any maximum $d$ set when $d=b(R)-1$. Since any vector and its complement are on opposite sides of the center in each coordinate, the sum of their distances to a vector $c$ that is at or within $\frac{1}{2}$ of the center in each coordinate is $b(R)$, which is even if $d$ is odd. If $c$ has half integral distance to lattice points, one of each complementary pair must have distance $d / 2$ or less to $c$, which proves the lemma.

Proof of Lemma 3. This proof is given in [2]. We note here that any maximum cardinality set $S$ with given diameter will always have elements with any fixed values of all but one coordinate that take on consecutive values of that coordinate. If we make these consecutive values an initial segment of the canonical ordering for every fixed value of the other coordinates, we cannot increase the diameter. The proof is an elaboration and verification of these two statements.

\section{Proofs of the Main Results}

In this section we prove Theorems 4 and 5 . The idea of the corresponding results of Kleitman and Fellows involved dividing the elements of $R$, according to their value of some component, into "outer" and "inner" elements. When $r=0$, the outer elements were those with the two extremal allowed values of that component. We could then deduce the desired conclusion for $R$ inductively from the same results for the two smaller sets, for $d<b(R)-1$.

The novelty here is the use of a different division of $R$ into inner and outer elements, when $d$ is $b(R)$ or larger. In particular, we separate the innermost two values of one component from the rest to prove Theorem 4 and the first in canonical order from the rest to prove Theorem 5 , for $d>b(R)-2+r$.

Proof of Theorem 4. Let $j$ be a component of our vectors which takes on an even number of values in $R$ and at least four of them. Let $R_{1 j}$ consist of those elements of $R$ whose $j$ th component is 0 or 1 , and $R_{2 j}=R-R_{1 j}$. Let $S$ be a canonical maximum $d$ set, and let $c$ be the center of $R$. Let $T$ be a set of radius $d / 2$ about $c$ when $d$ is even and about a point displaced by $\frac{1}{2}$ in the first coordinate from $c$ when $d$ is odd. Let the diameter of $R_{k j}$ be $t_{k j}$.

Let $S_{k j}$ be the intersection of $S$ with $R_{k j}$, and let its diameter be $d_{k j}$. We then have

$$
\begin{array}{rlrl}
d_{1 j} & \geq \min \left(d, t_{1 j}\right), \quad d_{2 j} & =d-2, \\
b\left(R_{1 j}\right) & \leq b(R), \quad b\left(R_{2 j}\right)=b(R)-2,
\end{array}
$$

and, therefore, if $d>b(R)-1$, then $d_{k j}>b\left(R_{k j}\right)-1$ for each value of $k$ 
We use the statement of this theorem as an induction hypothesis and apply it to each $\boldsymbol{R}_{k j}$, deducing here that the cardinality of each $S_{k j}$ is at most that of the corresponding $T_{k j}$, which upon addition proves that the cardinality of $S$ is at most that of $T$, as long as we can use the same $c$ for $T_{1 j}$ and $T_{2 j}$. The parities of $d_{1 j}$ and $d_{2 j}$ will be the same here, allowing the same center, unless $d_{1 j}<d$. In this case we may use the center of $T_{2 j}$ as the center for $T$ and still have $T_{1 j}=R_{1 j}$. This argument can be applied whenever any component has at least four distinct values in $R$. Otherwise $R$ is an $n$-cube, and we may use Lemma 1 .

The odd values of $d$ obeying $d<b(R)$ may be handled exactly like the even values: define $R_{3 j}$ to consist of the elements of $R$ whose $j$ th component is extremal in $R$; and $R_{4 j}=R-R_{3 j}$. We then observe that, upon extending the definitions of the $S$ 's $T$ 's, and $d$ 's, we have

$$
\begin{aligned}
d_{3 j} & =d-d_{j}+1, & d_{4 j} & =d, \\
b\left(R_{3 j}\right) & =b(R)-\left(d_{j}-1\right) / 2, & b\left(R_{4 j}\right) & =b(R)-1 .
\end{aligned}
$$

We may deduce that if $d<b(R)-1$, then $d_{3 j}<b\left(R_{3 j}\right)-1$ and $d_{4 j}<b\left(R_{4 j}\right)$. By induction we can conclude that the cardinality of $S$, which is the sum of that of $S_{3 j}$ and $S_{4 j}$, is at most the sum of that of $T_{3 j}$ and $T_{4 j}$ defined about the same center, which is the cardinality of $T$.

Proof of Theorem 5. Assume first that $d \geq b(R)-1+e(R)$. We proceed by induction on $e(R)$. Let $j$ be a component having an odd number of allowed values in $R$. Let $R_{5 j}$ consist of the elements of $R$ with the $j$ th component zero (which is the middle allowed value), let $R_{6 j}$ be $R-R_{5 j}$. Then we have, with the $S$ 's, $T$ 's, and $d$ 's defined as in the previous proof,

$$
\begin{aligned}
d_{5 j} & =\min \left(d, t_{5 j}\right), & d_{6 j} & =d-1, \\
b\left(R_{5 j}\right) & \leq b(R), & b\left(R_{6 j}\right) & =b(R), \\
e\left(R_{5 j}\right) & =e(R)-1, & & e\left(R_{6 j}\right)=e(R)-1, \\
o\left(R_{5 j}\right) & =o(R), & o\left(R_{6 j}\right) & =o(R)+1 .
\end{aligned}
$$

The cardinality of $S$ is that of $S_{5 j}$ plus that of $S_{6 j}$ which, by induction, is at most that of $T_{5 j}$ plus that of $T_{6 j}$. If $d_{5 j}=d$ the same centers may be chosen for both the $T_{k j}$ 's; otherwise the center of $T_{5 j}$ may be moved by $\frac{1}{2}$ without reducing its cardinality. In either case the cardinality of $S$ is at most that of $T$.

The $d$ even $d \leq b(R)-1$ case was treated by Kleitman and Fellows, and the odd case may be handled similarly. To do so, with $j$ as above, we let $R_{7 j}$ consist of the elements of $R$ with the extreme negative value of the $j$ th component, and let $R_{8 j}$ be the rest of $R$. We then have

$$
\begin{aligned}
d_{7 j} & =d-d_{j}, & d_{8 j} & =d, \\
b\left(R_{7 j}\right) & =b(R)-d_{j} / 2, & b\left(R_{8 j}\right) & =b(R), \\
e\left(R_{7 j}\right) & =e(R)-1, & e\left(R_{8 j}\right) & =e(R)-1 .
\end{aligned}
$$


The even and odd $d$ cases both follow by induction in parallel, as the parity of $d$ is the same as that of $d_{7 j}$ and $d_{8 j}$ here.

The $e(R)=0$ case is of course handled by Theorem 4 .

\section{Comments}

The results proven here resolve the Kleitman-Fellows conjecture except for certain "middle-size" values of the diameter. The number of such values is one less than the number, $e(R)$, of components whose range is odd among the vectors in $R$. For a fixed dimension $n$ of the vectors in $R$, the cases that are most unresolved are those for which all components have odd range, $e(R)=n$.

Intuitively, the $e(R)=n$ case is the one that seems easiest; we have proven that for small and large even $d$ the center of $R$ (and $\frac{1}{2}$ away from it for odd $d$ ) is the center of a maximum $d$ set of radius $d / 2$. It seems almost obvious that this statement is true for all $d$. Yet this is the case with the largest uncertain range here.

One conceivable way to prove the Kleitman-Fellows conjecture would be to show that every maximum $d$ set has radius $d / 2$. However, not every maximal $d$ set has radius $d / 2$, where maximal means no additional point may be added without increasing $d$, as the following three-dimensional example illustrates: $(000),(100)(010)(001)(110)(101)(011)(-100)(0-10)(00-1)$ has diameter 3 and is maximal though not maximum (in any $R$ large enough to contain it). It does not have radius $\frac{3}{2}$ about anywhere.

\section{References}

1. D. J. Kleitman, On a combinatorial conjecture of Erdös, J. Combin. Theory 1 (1966) 209-214.

2. D. J. Kleitman and M. Fellows, Radius and diameter in Manhattan lattices, Discrete Math., to appear. 